\title{
The Role of Thiol/Disulphide Homeostasis in Anthracycline Associated Cardiac Toxicity
}

\author{
Mustafa Topuz, ${ }^{1}$ MD, Omer ŞEn,,${ }^{1} \mathrm{MD}$, Mehmet Kaplan, ${ }^{1} \mathrm{MD}$, Oguz AKKus, ${ }^{1} \mathrm{MD}$, \\ Ozcan Erel, ${ }^{2} \mathrm{PhD}$, and Mustafa Gur, ${ }^{1} \mathrm{PhD}$
}

\begin{abstract}
SUMMARY
The aim of the present study was to evaluate whether the baseline thiol/disulfide state can predict the occurrence of anthracycline induced cardiac toxicity. A total of 186 cancer patients receiving anthracycline (doxorubicin)-based chemotherapy were enrolled. All patients underwent 2-dimensional (2D) speckle tracking echocardiography (STE) to determine their left ventricular ejection fraction (LVEF) and blood samples for measuring thiol forms were obtained before treatment and 4 weeks after completion of the chemotherapy. The mean dose of doxorubicin exposure was $255 \pm 39.2$ $\mathrm{mg} / \mathrm{m}^{2}$. Baseline native thiol was found to be lower whereas baseline disulfide and the disulfide/total thiol ratio were found to be higher in patients who had a decrease in LVEF after anthracycline therapy. Also, the amount of decrease in LVEF was well correlated with the delta value of the thiol forms. Logistic regression analysis revealed that changes in BNP and global longitudinal strain (GLS), baseline level of native thiol, disulfide, and the disulfide/total thiol ratio were strong predictors for a decrease in LVEF.

The thiol/disulfide pathway may be a factor for predicting chemotherapy-induced cardiac toxicity as one of the oxidative stress mechanisms. (Int Heart J 2017; 58: 69-72)
\end{abstract}

Key words: Oxidative stress, Anthracyclines, Cardiomyopathy

$\mathrm{A}$

lthough chemotherapeutic treatment has led to a better prognosis for cancer patients, some treatments can increase cardiovascular morbidity because of cardiac toxicity. ${ }^{1,2)}$ Chemotherapy-induced cardiac toxicity is complex but involves dose-related myocardial cell death and loss of myocardial mass, leading to progressive cardiac remodeling and cardiac dysfunction due to oxidative and nitrosative stress. ${ }^{3,4)}$ Anthracycline, an effective chemotherapeutic agent, is a well-known example of a cardiotoxic chemotherapeutic agent. $^{5,6)}$ Thus, the evaluation of cardiac functions before, during, and after treatment containing anthracycline is clinically essential.

When the generation of intracellular reactive oxygen species (ROS) exceeds the local antioxidant capacity, multiple intracellular adaptive mechanisms are up-regulated to effort tissue protection and prevent progression to apoptosis and/or necrosis. One of the protective mechanisms against oxidative cell damage is the thiol groups of cellular protein. ${ }^{7)}$ Thiols are a class of organic compounds that contain a sulfhydryl group $(-\mathrm{SH})$ composed of a sulfur atom and a hydrogen atom attached to a carbon atom. ${ }^{8)}$ Thiols (RSH) can undergo oxidation reaction via oxidants and form disulfide (RSSR) bonds. ${ }^{9)}$ The formed disulfide bonds can again be reduced to thiol groups; so, dynamic thiol-disulfide homeostasis is maintained. ${ }^{10)}$ This homeostasis has a critical role in antioxidant protection, de- toxification, signal transduction, apoptosis, regulation of enzymatic activity, and transcription factors and cellular signaling mechanisms. ${ }^{11,12)}$

Although thiol/disulfide homeostasis has been increasingly studied in many disorders, no studies thus far have investigated thiol/disulfide homeostasis in cancer patients who suffered from anthracycline-induced cardiotoxicity. ${ }^{13,14)}$ Thus, we aimed to evaluate whether thiol/disulfide homeostasis may plav a role in the protection of myocardial cells from anthracycline-induced toxicity as a novel oxidative stress parameter.

\section{Methods}

A total of 186 cancer patients treated with anthracycline (doxorubicin) based chemotherapy due to breast cancer ( $n=$ $120)$, Hodgkin's lymphoma $(n=18)$, and non-Hodgkin's lymphoma $(n=48)$ were enrolled. Patient demographic and baseline characteristics were recorded. Patients with systolic heart failure ( $\mathrm{LVEF} \leq 50 \%$ ), coronary artery disease, acute or chronic infectious diseases, acute or chronic renal failure, acute or chronic hepatic failure, chronic inflammatory and autoimmune diseases, treatment with angiotensin converting enzyme (ACE)/angiotensin receptor blockers (ARB) and/or $\beta$-blocker and/or statin, and concomitant dexrazoxane therapy in addition

From the ${ }^{1}$ Department of Cardiology, Adana Numune Education and Research Hospital, Adana, and ${ }^{2}$ Department of Biochemistry, Yildırım Beyazıt University, Ankara, Turkey.

Address for correspondence: Mustafa Topuz, MD, Department of Cardiology, University of Health Sciences, Adana Numune Education and Research Hospital, Süleyman Demirel Bulvah, Kurttepe mevki, Cukurova, Adana 01150, Turkey. E-mail: mtpuz@ hotmail.com

Received for publication March 16, 2016. Revised and accepted July 4, 2016.

Released in advance online on J-STAGE January 17, 2017.

All rights reserved by the International Heart Journal Association. 
to anthracycline were excluded. All patients provided written informed consent and the study protocol was approved by the local ethics committee.

Echocardiographic examination: All conventional echocardiographic examinations were performed in the left lateral decubitus position by one cardiologist in accordance with current guidelines. ${ }^{15)}$ The average values of all echocardiographic measurements were obtained after 3 consecutive cardiac cycles. The LVEF was calculated according to the modified Simpson's rule. ${ }^{16)}$ The myocardial performance index (MPI) was determined using Doppler time intervals measured from mitral inflow and left ventricular outflow Doppler tracings.

Two-dimensional (2-D) strain imaging was used to determine LV myocardial deformation before and after anthracycline therapy. For the assessment of global longitudinal, circumferential, and radial speckle-tracking strain (GLS\%, GCS\%, and GRS\%), standard 2D ultrasound images at the parasternal mid-ventricular short-axis view (at the level of the papillary muscles), the apical long-axis view, and 2-chamber and 4-chamber views were used with a frame rate between 60 and 80 fps (EpiQ 7, Affiniti 70 ultrasound system, USA) as previously described. ${ }^{17-19)}$ After manual tracing of the endocardial borders, the software automatically traced the region of interest including the entire myocardial wall. In this process, every view of the left ventricle was divided into 6 segments. To optimize tracking, the width of the region of interest was adjusted if necessary.

The echocardiographic measurements of 30 randomly selected patients were re-evaluated by another experienced echocardiographer who was blinded to the clinical data to calculate intra and intraobserver variability on two consecutive days. Intraobserver variability was found to be $2.2 \%$ and the interobserver variability was $2.7 \%$.

Laboratory tests: Serum samples were collected by a peripheral venous route before the chemotherapy and after 4 weeks. Hematologic parameters were measured from tripotassium ethylenediaminetetraacetic acid-based anticoagulated blood samples and assessed using a Sysmex K-1000 (Block Scientific, Bohemia, NY, USA) auto analyzer within 30 minutes of sampling. The serum was then separated from the cells by centrifugation at $3000 \mathrm{rpm} / 10$ minutes for biochemical (Roche Diagnostics, Indianapolis, IN, USA) and thiol analyses. The serum samples for thiol were immediately stored on ice at $-41^{\circ} \mathrm{C}$ until analyzed. Total thiol (-S-S- + - SH) consists of native and reduced thiol. First, we used sodium borohydride as a reductant solution (10 $\mu \mathrm{L})$ to reduce disulfide bonds (-S-S-) to functional thiol groups. Similarly, after dissolving sodium chloride in $1000 \mathrm{~mL}$ of water-methanol solution, we obtained another reduction solution $(10 \mu \mathrm{L})$ for determining native thiol $(-\mathrm{SH})$ content. The disulfide parameter is a value which can be calculated automatically as half of the difference of the two measured values. After calculation of the main parameters (native thiol, total thiol, and disulfide numerical values) the disulfide/total thiol (-S-S-)/(-S-S- + -SH) ratio was obtained. The intra-assay coefficient of variation was evaluated by performing 15 repetitions in a single analytical run, using the serum of healthy subjects. The intra-assay coefficient of variation was found to be $1.5 \%$. The inter-assay coefficient of variation was evaluated in triplicate (on 3 different dispensing cycles) in 5 different analytical runs using the serum of healthy subjects. The inter-assay coefficient of variation was $2.1 \%$.
Statistical analysis: $\quad$ SPSS statistical software (version 16.0, SPSS, USA) was used. Variables were investigated using visual (histograms and probability plots) and analytical methods (Kolmogorov-Smirnov test) to determine whether they were normally distributed and are expressed as the mean \pm standard deviation (SD) or median and interquartile range (IQR, range from the 25 th to the 75 th percentile). The Mann-Whitney $U$ test was used for the comparison of two groups with a nonnormal distribution of variables and the chi-square test was used to compare qualitative data for both initial and control values. Spearman correlation was performed to evaluate the association between changes in LVEF with other study parameters. Multivariate logistic regression analysis was used to identify predictors for reduced LVEF. Intraobserver and interobserver variability were measured by the coefficient of repeatability $(\mathrm{COR}){ }^{20)}$ A $P$ value $<0.05$ was accepted to be statistically significant.

\section{Results}

A total of 186 cancer patients (123 females, 63 males) with a mean age of $55.7 \pm 10.8$ years and who received combination anthracycline-based chemotherapy (as doxorubicin, mean dose $255.2 \pm 39.2 \mathrm{mg} / \mathrm{m}^{2}$, with cyclophosphamide in those with a diagnosis of breast cancer, and with cyclophosphamide, vincristine, and prednisone in those with a diagnosis of Hodgkin's and non-Hodgkin's lymphoma) were included in the present study. No clinical cardiac events were observed during the study period.

The mean LVEF was $60.6 \pm 3.3 \%$. At the end of chemotherapy, LVEF was decreased in 36 patients. We determined the criteria for a decrease in LVEF as a $\geq 5 \%$ reduction compared to its baseline value. Those subjects with a decline in LVEF (mean LVEF, $52.0 \pm 5.08 \%$ ) were compared to those with no change in LVEF (mean LVEF, $60.4 \pm 3.03 \%$ ). The baseline characteristics of the 2 groups before chemotherapy are listed in Supplemental Table I.

Patients who experienced a decline in LVEF had lower baseline native thiol (-SH) but higher baseline disulfide (-S-S-) and disulfide/total thiol (-S-S-)/(-S-S- + -SH) ratio than patients without a decrease in LVEF (Supplemental Figure 1, Supplemental Table I).

At the end of chemotherapy, native thiol was decreased, and the disulfide level and disulfide/total thiol ratio were increased in all study patients (Supplemental Table II). The change ratio $(\Delta)$ was calculated first subtracting the baseline value of the study parameters from the one month value and then divided by the baseline value. The $\Delta$ values of native thiol, disulfide, and the disulfide/total thiol ratio were more altered in patients without a change in LVEF than in those patients who had a reduction in LVEF $(P<0.001$ for both $\Delta$ native thiol and $\Delta$ disulfide, and $P=0.011$ for the $\Delta$ disulfide/total thiol ratio). Also, a decrease in native thiol and an increase in the disulfide level after anthracycline therapy were associated with preserved LVEF in the study patients (Supplemental Figures 2 and 3).

In TTE examination, although MPI did not show a significant change, all of the GLS, GCS, and GRS parameters had deteriorated during the early phase of the anthracycline treatment (Supplemental Table II). The intraobserver intraclass co- 
efficients for GLS, GCS, and GRS were 0.91 (CV 2.6\%), 0.93 (CV 2.9\%), and 0.92 (CV 3.0\%), respectively. The interobserver intraclass coefficients for GLS, GCS, and GRS were 0.89 (CV 3.6\%), 0.87 (CV 4.5\%), and 0.85 (CV 4.7\%), respectively.

In the correlation analysis, the $\Delta$ value of LVEF had significant correlations with the $\Delta$ values of GLS, native thiol, disulfide, and disulfide/total thiol ratio, and BNP (Supplemental Table III).

Multivariable logistic regression analysis revealed changes in BNP and global longitudinal strain (GLS) in STE, initial levels of native thiol and disulfide, and disulfide/total thiol ratio were strong predictors for a decrease in LVEF after chemotherapy (Supplemental Table IV).

\section{Discussion}

To the best of our knowledge, this is the first study that showed that thiol/disulfide homeostasis may play a role in chemotherapy-induced cardiac toxicity in cancer patients treated with anthracycline based chemotherapy.

In light of previous studies that indicated the pathophysiologic role of oxidative stress in cancer, we hypothesized that there might be impaired thiol/disulfide homeostasis in cancer patients who have anthracycline-induced cardiotoxicity. Oxidative stress has attracted the interest of clinicians for a long time and it has been shown that it has a critical role in the progress of various diseases such as type 1 diabetes mellitus, cancer, atherosclerosis, and hypertension. ${ }^{21-24)}$ Both excessive oxidative stress and inadequate defence of cardiomyocytes can also play a role in the development of early onset of chemotherapy-induced cardiotoxicity. ${ }^{3)}$

Myocardial cells, a metabolically active tissue, have an elegant system of antioxidant defenses and cell repair mechanisms against oxidative and nitrosative stress which can cause cell necrosis and/or apoptosis. ${ }^{25-27)}$ The cardiotoxic mechanism of anthracycline may involve dose-related myocardial cell death, probably due to an impairment of reparatory and homoeostatic mechanisms after exposure to chemotherapy., ${ }^{3,4)}$ Thiol contains a sulfhydryl (-SH) group and plays a critical role in the prevention of oxidative stress in cells and its level can be altered during proliferation or apoptosis at the cellular level. ${ }^{28,29)}$ As primary targets of oxygen radicals, the $-\mathrm{SH}$ groups of sulfur containing amino acids such as cysteine and methionine in proteins are oxidized and return to reversible disulfide bonds. The structural and functional alterations in cell proteins begin after loss of these thiol groups. ${ }^{30,31)}$ Thiol groups attempting to prevent the devastating effects of free radicals may reduce their plasma and tissue levels during those interactions. ${ }^{32)}$ In fact, similar to previous studies, we found that the native thiol level was decreased whereas the disulfide level and disulfide/total thiol ratio were increased in all study patients as a result of oxidative damage. ${ }^{30,33,34)}$ Based on these findings, we can speculate that thiol/disulfide homeostasis was also altered in cancer patients during anthracycline therapy.

Moreover, the alteration in thiol forms was greater in patients who had preserved LVEF than in those patients with a decrease in LVEF. In this respect, we may also suggest that the thiol/disulfide homeostasis pathway could contribute more to counter the oxidative damage in the normal EF group as we demonstrated in Supplemental Figure 3. Also, we found that patients who had higher native thiol levels at baseline seemed to be less affected by anthracycline associated cardiac toxicity. We observed significant correlations between the initial native thiol level and the disulfide level with the differences in LV global longitudinal strain. Based on our results, we believe that baseline thiol forms may be predictors of patients who in the future may suffer cardiotoxicity after anthracycline therapy.

We evaluated the LVEF with 2D STE and found significant correlations between the initial native thiol and disulfide levels with the differences in LV GLS value. Although a decrease in LVEF may reflect myocardial injury and the first sign of cardiac toxicity in cancer patients, transthoracic echocardiography or nuclear scans are not very sensitive in predicting which patient eventually develops cardiac failure. ${ }^{35}$ Reductions in myocardial deformation parameters in STE could be a sign of subclinical myocardial changes from cancer therapy and occur prior to any change in LVEF as assessed by conventional echocardiography. ${ }^{18,19)}$ It was previously showed that GLS could be used as an early marker of chemotherapy-induced cardiac toxicity. ${ }^{18)}$ Moreover, GLS values can also predict later reductions in $\mathrm{EF}$, and is more powerful at predicting the effect of chemotherapy-induced toxicity than GL strain rate values. ${ }^{36)}$ In our study, we also found changes in BNP levels may predict future cardiac toxicity in logistic analyses. Actually, a lot of cardiac biomarkers such as BNP, N-terminal pro-BNP (NTproBNP), and cardiac troponin $\mathrm{T}$ in addition to LVEF have been studied before and showed that all of these markers may have a role in chemotherapy-induced cardiotoxicity and may be useful during the follow-up period to enable early detection. ${ }^{37-41)}$ There are several limitations with respect to measuring cardiac biomarkers levels since other diseases and conditions such as age, heart failure, or ischemia may also cause abnormal levels of BNP and troponin T.

Our study patients were similar according to baseline characteristics including age, hypertension, and diabetes mellitus which can alter plasma thiol and we also excluded patients with a history of CAD ${ }^{32}$ After adjusting for all of these factors, patients with a reduced LVEF had a lower baseline level of native thiol but a higher disulfide level and disulfide/total thiol ratio than those with no change in LVEF. All of these parameters were found to be significant predictors for a decline in LVEF in logistic analyses. We can speculate that higher baseline native thiol levels could mean higher protection from oxidative stress, whereas a higher disulfide level or lower baseline thiol level could indicate a patient is more prone to the structural and functional alterations in cell proteins and apoptosis.

Conclusion: Our findings concerning thiol may provide more objective data for physicians to detect which patients are at high risk for cardiac toxicity before chemotherapy. The ability and usability of the thiol/disulfide ratio for anthracycline associated cardiac toxicity may lead to new recommendations for monitoring cancer patients during and after chemotherapy.

Limitations: Long-term follow-up of patients is needed because the full spectrum of cardiotoxicity frequently does not become apparent until months or even years after the initial cancer treatment. For this reason, patients could be followedup for a longer period of time. Also, both other oxidative stress parameters or new biomarkers as well as imaging modalities should be studied in future trials to further validate our findings. 


\section{REFERENCES}

1. Borchmann P, Eichenauer DA, Engert A. State of the art in the treatment of Hodgkin lymphoma. Nat Rev Clin Oncol 2012; 9: 450-9. (Review)

2. Darby S, McGale P, Correa C, et al. Effect of radiotherapy after breast-conserving surgery on 10-year recurrence and 15-year breast cancer death: meta-analysis of individual patient data for 10,801 women in 17 randomised trials. Lancet 2011; 378: 170716. (Review)

3. Eschenhagen T, Force T, Ewer MS, et al. Cardiovascular side effects of cancer therapies: a position statement from the Heart Failure Association of the European Society of Cardiology. Eur J Heart Fail 2011; 13: 1-10. (Review)

4. Sawyer DB, Peng X, Chen B, Pentassuglia L, Lim CC. Mechanisms of anthracycline cardiac injury: can we identify strategies for cardioprotection? Prog Cardiovasc Dis 2010; 53: 105-13. (Review)

5. Verdecchia A, Francisci S, Brenner H, et al. Recent cancer survival in Europe: a 2000-02 period analysis of EUROCARE- 4 data. Lancet Oncol 2007; 8: 784-96.

6. Koitabashi N, Ohyama Y, Tateno R, et al. Reversible cardiomyopathy after epirubicin administration. Int Heart J 2015; 56: 466-8.

7. Thomas JA, Poland B, Honzatko R. Protein sulfhydryls and their role in the antioxidant function of protein S-thiolation. Arch Biochem Biophys 1995; 319: 1-9. (Review)

8. Sen CK, Packer L. Thiol homeostasis and supplements in physical exercise. Am J Clin Nutr 2000; 72: 653S-69S. (Review)

9. Cremers CM, Jakob U. Oxidant sensing by reversible disulfide bond formation. J Biol Chem 2013; 288: 26489-96. (Review)

10. Jones DP, Liang Y. Measuring the poise of thiol/disulfide couples in vivo. Free Radic Biol Med 2009; 47: 1329-38.

11. Biswas S, Chida AS, Rahman I. Redox modifications of proteinthiols: emerging roles in cell signaling. Biochem Pharmacol 2006; 71: 551-64.

12. Circu ML, Aw TY. Reactive oxygen species, cellular redox systems, and apoptosis. Free Radic Biol Med 2010; 48: 749-62. (Review)

13. Go YM, Jones DP. Cysteine/cystine redox signaling in cardiovascular disease. Free Radic Biol Med 2011; 50: 495-509. (Review)

14. Prabhu A, Sarcar B, Kahali S, et al. Cysteine catabolism: a novel metabolic pathway contributing to glioblastoma growth. Cancer Res 2014; 74: 787-96.

15. Quiñones MA, Otto CM, Stoddard M, Waggoner A, Zoghbi WA Recommendations for quantification of Doppler echocardiography: a report from the Doppler Quantification Task Force of the Nomenclature and Standards Committee of the American Society of Echocardiography. J Am Soc Echocardiogr 2002; 15: 167-84.

16. Lang RM, Bierig M, Devereux RB, et al. Recommendations for chamber quantification. Eur J Echocardiogr 2006; 7: 79-108. (Review)

17. Thavendiranathan P, Poulin F, LimD K, Plana JC, Woo A, Marwick TH. Use of myocardial strain imaging by echocardiography for the early detection of cardiotoxicity in patients during and after cancer chemotherapy: a systematic review. J Am Coll Cardiol 2014; 63: 2751-68. (Review)

18. Urheim S, Edvardsen T, Torp H, Angelsen B, Smiseth OA. Myocardial strain by Doppler echocardiography. Validation of a new method to quantify regional myocardial function. Circulation 2000; 102: 1158-64.

19. Kim YH, Choi J, Kim SH, Kim DH, Ahn JC, Song WH. Patterns of decrease in multidirectional myocardial deformations in patients with fluctuating left ventricular ejection fraction. Int Heart $\mathrm{J}$ 2014; 55: 319-25.

20. Bland JM, Altman DG. Statistical methods for assessing agreement between two methods of clinical measurement. Lancet 1986; 1: 307-10.

21. Ateş I, Kaplan M, Yuksel M, et al. Determination of thiol/disulphide homeostasis in type 1 diabetes mellitus and the factors associated with thiol oxidation. Endocrine 2016; 51: 47-51.
22. Dirican N, Dirican A, Sen O, et al. Thiol/disulfide homeostasis: A prognostic biomarker for patients with advanced non-small cell lung cancer? Redox Rep 2016; 21: 197-203.

23. Liu WJ, Wang T, Wang B, et al. CYP2C8-derived epoxyeicosatrienoic acids decrease oxidative stress-induced endothelial apoptosis in development of atherosclerosis: Role of Nrf2 activation. J Huazhong Univ Sci Technolog Med Sci 2015; 35: 640-5.

24. Ates I, Ozkayar N, Altay M, et al. Is disulphide/thiol ratio related to blood pressure in masked hypertension? Clin Exp Hypertens 2016; 38: 150-4

25. Winterbourn CC. Reconciling the chemistry and biology of reactive oxygen species. Nat Chem Biol 2008; 4: 278-86.

26. Thomas JA, Poland B, Honzatko R. Protein sulfhydryls and their role in the antioxidant function of protein S-thiolation. Arch Biochem Biophys 1995; 319: 1-9. (Review)

27. Reed DJ. Glutathione: toxicological implications. Annu Rev Pharmacol Toxicol 1990; 30: 603-31. (Review)

28. Nkabyo YS, Ziegler TR, Gu LH, Watson WH, Jones DP. Glutathione and thioredoxin redox during differentiation in human colon epithelial (Caco-2) cells. Am J Physiol Gastrointest Liver Physiol 2002; 283: G1352-9.

29. Di Simplicio P, Cacace MG, Lusini L, Giannerini F, Giustarini D, Rossi R. Role of protein-SH groups in redox homeostasis--the erythrocyte as a model system. Arch Biochem Biophys 1998; 355 : $145-52$.

30. Dean RT, Fu S, Stocker R, Davies MJ. Biochemistry and pathology of radical-mediated protein oxidation. Biochem J 1997; 324 : 1-18. (Review)

31. Ziegler DM. Role of reversible oxidation-reduction of enzyme thiols-disulfides in metabolic regulation. Annu Rev Biochem 1985; 54: 305-29. (Review)

32. McCord JM. Human disease, free radicals, and the oxidant/antioxidant balance. Clin Biochem 1993; 26: 351-7. (Review)

33. Kundi H, Ates I, Kiziltunc E, et al. A novel oxidative stress marker in acute myocardial infarction; thiol/disulphide homeostasis. Am J Emerg Med 2015; 33: 1567-71.

34. Kundi H, Erel Ö, Balun A, et al. Association of thiol/disulfide ratio with syntax score in patients with NSTEMI. Scand Cardiovasc J 2015; 49: 95-100.

35. Ewer MS, Ali MK, Mackay B, et al. A comparison of cardiac biopsy grades and ejection fraction estimations in patients receiving Adriamycin. J Clin Oncol 1984; 2: 112-7.

36. Negishi K, Negishi T, Hare JL, Haluska BA, Plana JC, Marwick $\mathrm{TH}$. Independent and incremental value of deformation indices for prediction of trastuzumab-induced cardiotoxicity. J Am Soc Echocardiogr 2013; 26: 493-8.

37. Daugaard G, Lassen U, Bie P, et al. Natriuretic peptides in the monitoring of anthracycline induced reduction in left ventricular ejection fraction. Eur J Heart Fail 2005; 7: 87-93.

38. Aggarwal S, Pettersen MD, Bhambhani K, Gurczynski J, Thomas $\mathrm{R}$, L'Ecuyer T. B-type natriuretic peptide as a marker for cardiac dysfunction in anthracycline-treated children. Pediatr Blood Cancer 2007; 49: 812-6.

39. Cardinale D, Sandri MT. Role of biomarkers in chemotherapy-induced cardiotoxicity. Prog Cardiovasc Dis 2010; 53: 121-9. (Review)

40. Lipshultz SE, Miller TL, Scully RE, et al. Changes in cardiac biomarkers during doxorubicin treatment of pediatric patients with high-risk acute lymphoblastic leukemia: associations with longterm echocardiographic outcomes. J Clin Oncol 2012; 30: 1042-9.

41. Takeishi Y. Biomarkers in heart failure. Int Heart J 2014; 55: 47481. (Review)

\section{SuPPlemental FiLes}

Supplemental Tables I, II, III, IV

Supplemental Figures 1, 2,3

Please see supplemental files;

https://www.jstage.jst.co.jp/article/ihj/58/1/58_16-124/_article/supplement 